\title{
Mechanical Properties
}

of Viscoelastic Media

Erik H. Clayton ${ }^{1}$

e-mail: clayton@wustl.edu

\section{Ruth J. Okamoto}

Department of Mechanical Engineering and Materials Science, Washington University in St. Louis, One Brookings Drive,

Campus Box 1185,

Saint Louis, M0 63130

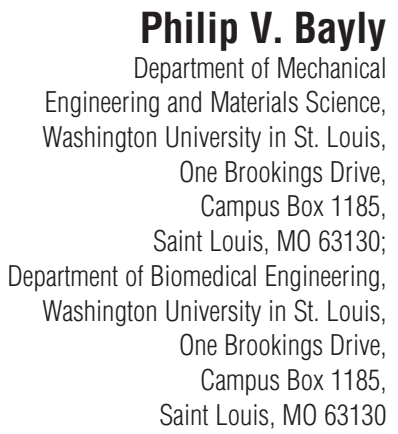

Philip V. Bayly

Engineering and Materials Science,

Oniversity in St. Louls, e Brookings Drive,

Campus Box 1185

iomedical Engineering, Campus Box 1185,

Saint Louis, M0 63130

\section{by Local Frequency Estimation of Divergence-Free Wave Fields}

\begin{abstract}
Magnetic resonance elastography (MRE) is an imaging modality with which mechanical properties can be noninvasively measured in living tissue. Magnetic resonance elastography relies on the fact that the elastic shear modulus determines the phase velocity and, hence the wavelength, of shear waves which are visualized by motion-sensitive MR imaging. Local frequency estimation (LFE) has been used to extract the local wavenumber from displacement wave fields recorded by MRE. LFE -based inversion is attractive because it allows material parameters to be estimated without explicitly invoking the equations governing wave propagation, thus obviating the need to numerically compute the Laplacian. Nevertheless, studies using LFE have not explicitly addressed three important issues: (1) tissue viscoelasticity; (2) the effects of longitudinal waves and rigid body motion on estimates of shear modulus; and (3) mechanical anisotropy. In the current study we extend the LFE technique to (1) estimate the (complex) viscoelastic shear modulus in lossy media; (2) eliminate the effects of longitudinal waves and rigid body motion; and (3) determine two distinct shear moduli in anisotropic media. The extended LFE approach is demonstrated by analyzing experimental data from a previouslycharacterized, isotropic, viscoelastic, gelatin phantom and simulated data from a computer model of anisotropic (transversely isotropic) soft material.
\end{abstract} [DOI: $10.1115 / 1.4023433]$

Keywords: magnetic resonance elastography, mechanical characterization, viscoelasticity, isotropy, transverse isotropy, anisotropy, tissue

\section{Introduction}

Magnetic resonance elastography (MRE) is a recentlydeveloped experimental technique for noninvasively probing the dynamic shear modulus of soft biological tissue in living subjects [1-5]. Magnetic resonance elastography is performed by inducing steady-state harmonic shear waves within a region of interest via a mechanical actuator and a standard magnetic resonance imaging (MRI) scanner, equipped with a motion-encoding imaging sequence, is used to capture spatiotemporal images of the propagating waves. Regional estimates of tissue shear modulus are made by a local analysis of the wave field using wavelength measurement $[1,4]$, direct inversion of the wave equation [3,5], or inverse finite element (FE) methods [6]. The utility of MRE is that mechanical properties can be used to identify baseline properties of healthy tissues or to identify and assess local or regional changes in tissue properties associated with injury or disease.

Magnetic resonance elastography has been used to characterize the components of the viscoelastic shear modulus in brain, liver, skeletal muscle, and other biologic tissue [7-17]. Nonetheless, disparity in measurements made by different laboratories suggests uncertainties attributable to methodology. For example, a range of shear moduli have been reported in brain tissue [18]. Differences in experimental setup, data collection, and data analysis may be contributing factors. Until the uncertainties in material properties have been quantified, explained, and reduced, researchers and clinicians will be hesitant to rely on MRE.

\footnotetext{
Corresponding author.

Contributed by the Bioengineering Division of ASME for publication in the Journal of BiomeChANICAL ENGINEERING. Manuscript received October 1, 2012; final manuscript received January 14, 2013; accepted manuscript posted January 18, 2013; published online February 7, 2013. Editor: Victor H. Barocas.
}

The variability in the estimated mechanical properties may be attributed to the material model assumed and the mathematical scheme used to invert the governing equations of motion. As a first approximation, an isotropic material model is commonly assumed for biological tissue. Direct inversion (least-squares fitting of parameters to wave equations), inverse finite element methods, and local frequency estimation (LFE) have different advantages and disadvantages. Estimates of mechanical properties based on LFE have reduced errors attributable to numerical differentiation, but can be corrupted by rigid body motion and dilatational waves in displacement data. Analysis of raw displacement data is challenging because shear and longitudinal wave motion are intrinsically coupled through loading and boundary conditions [19], but the two wave speeds differ by several orders of magnitude. In previous work [17], we have suggested that this type of error can be remedied by applying LFE to the curl of the displacement field, which requires only a single spatial derivative in each direction. The application of LFE to viscoelastic or anisotropic materials has not yet been verified.

In this study, we develop an extended LFE inversion scheme and verify it in a cylindrical geometry in which axial displacement of a central rod induces radially propagating shear waves. We use both a closed-form analytical wave field and experimental data from a gelatin-based tissue analog [20] to demonstrate our distortion-based LFE approach on viscoelastic isotropic media. Wave propagation in a transversely isotropic material is simulated by the finite element (FE) model and used to verify the extension of our LFE method to anisotropic materials.

\section{Methods}

Local frequency estimation (LFE) [21] has been used to locally extract the wavenumber $\kappa=|\overline{\mathbf{k}}|$ from displacement wave fields in 
order to estimate the elastic shear modulus [4,5]. LFE-based inversion is attractive because it allows material parameters to be estimated without explicitly invoking the coupled or uncoupled equations of motion and, consequentially, eliminates the need to numerically compute second or third-order spatial derivatives. LFE applied to displacement fields is challenging in practice since dilatational waves and zero-strain rigid body motion can corrupt wavelength estimates. In prior studies, these effects have been treated by either applying aggressive filters to displacement data [5] or by applying the curl operator to equations of motion and inverting equations containing third-order spatial derivatives [7].

In this study, viscoelastic properties at multiple frequencies were obtained by local frequency estimation applied to distortion fields estimated from the curl of displacement. The shear wave velocity depends only on the shear modulus and density of the media through which it is traveling (see Eq. 2(b)). Application of the LFE to the distortion field avoids artifacts attributed to rigid body motion and dilatational waves in displacement data.

Implicit in existing LFE-based inversion formulations $[4,5,22]$ is the wave phase velocity relation $c=\omega / \kappa$, which permits elastic material property reconstruction. In this study, we use the equation describing planar shear wave propagation in an isotropic lossy medium to extract viscoelastic shear moduli $[23,24]$. These estimates are subject to the validity of the basic assumptions of MRE: (1) that strains are small, so that the equations of linear elasticity and viscoelasticity apply; and (2) that the medium is "locally homogeneous" [5] so that material properties do not vary significantly within a single wavelength or a few voxels. Under these conditions, in an isotropic material, the shear wave velocity will depend only on (complex) shear modulus and density. Finally, we apply LFE to directionally-filtered wave fields to extract anisotropic material properties.

2.1 Divergence-Free Wave Propagation in Elastic Media. The general equations governing wave propagation in a linear elastic, isotropic, homogeneous material are

$$
\rho \frac{\partial^{2} u_{k}}{\partial t}=\mu \nabla^{2} u_{k}+(\lambda+\mu) \frac{\partial}{\partial x_{k}}(\nabla \cdot \overline{\mathbf{u}})
$$

where $\lambda$ and $\mu$ are the Lamé constants and $\rho$ is the material density [23]. The dilatational (irrotational) and distortional (equivoluminal, divergence-free, or shear) deformation modes can be decoupled by applying the divergence and curl to Eq. (1)

$$
\begin{gathered}
\frac{\partial^{2}}{\partial t^{2}}(\nabla \cdot \mathbf{u})=\frac{\lambda+2 \mu}{\rho} \nabla^{2}(\nabla \cdot \overline{\mathbf{u}}) \\
\frac{\partial^{2}}{\partial t^{2}}(\nabla \times \overline{\mathbf{u}})=\frac{\mu}{\rho} \nabla^{2}(\nabla \times \overline{\mathbf{u}})
\end{gathered}
$$

respectively $[19,23,24]$. Waves of dilatation travel at a speed of $c_{1}=\sqrt{(\lambda+2 \mu) / \rho}$ and waves of distortion at $c_{2}=\sqrt{\mu / \rho}$. The application of LFE to the curl of the displacement field, rather than to the displacement field itself, eliminates the effects of longitudinal waves and rigid body motion.

The geometry of the specimens used in this study lends well to analysis in a polar coordinate system. Hence, the curl in polar coordinates

$$
\begin{aligned}
\nabla \times \overline{\mathbf{u}}(t)= & \left(\frac{1}{r} \frac{\partial u_{z}(t)}{\partial \theta}-\frac{\partial u_{\theta}(t)}{\partial z}\right) \overline{\mathbf{e}}_{\mathbf{r}}+\left(\frac{\partial u_{r}(t)}{\partial z}-\frac{\partial u_{z}(t)}{\partial r}\right) \overline{\mathbf{e}}_{\theta} \\
& +\frac{1}{r}\left(\frac{\partial\left(r u_{\theta}(t)\right)}{\partial r}-\frac{\partial u_{r}(t)}{\partial \theta}\right) \overline{\mathbf{e}}_{\mathbf{z}}
\end{aligned}
$$

Since we observe that $u_{r}(t) \approx u_{\theta}(t) \approx \partial u_{z}(t) / \partial \theta \approx 0$ and $u_{z}(t) \neq 0$, let

$$
\Gamma(t)=-\frac{\partial u_{z}(t)}{\partial r}
$$

be the in-plane distortional wave component utilized in this study. We also denote $\Gamma(\omega)$ as the fundamental harmonic of the Fourier transformed distortion field $\Gamma(t)$.

2.2 Viscoelastic Parameter Estimation. In viscoelastic materials, by using the correspondence principle, the elastic shear modulus $\mu$ in Eq. (2b) may be replaced by the complex viscoelastic shear modulus $G^{*}=G^{\prime}+i G^{\prime \prime}$. A harmonic plane wave of arbitrary amplitude $A$ and frequency $\omega$ traveling in an isotropic viscoelastic material is described by the following solution:

$$
u(x, t)=A e^{-\alpha x} \exp [i(\omega t-\kappa x)]
$$

where $\kappa$ is the wavenumber and $\alpha$ is the attenuation [25]. We note that the distortion $\Gamma$ is of the same form as Eq. (5) and that the distortion field satisfies the equation governing shear wave propagation (Eq. (2b)). By substituting the preceding wave field solution into the viscoelastic shear wave equation for steady-state harmonic motion we obtain the following expression:

$$
\left[\begin{array}{cc}
\kappa^{2}-\alpha^{2} & 2 \alpha \kappa \\
-2 \alpha \kappa & \kappa^{2}-\alpha^{2}
\end{array}\right]\left\{\begin{array}{c}
G^{\prime} \\
G^{\prime \prime}
\end{array}\right\}=\left\{\begin{array}{c}
\rho \omega^{2} \\
0
\end{array}\right\}
$$

in which the storage $G^{\prime}$ and loss $G$ moduli are in terms of $\kappa, \alpha$, material density $\rho$, and driving frequency $\omega$.

The parameter $\alpha$ can be estimated from the rate of exponential decay of wave peaks (logarithmic decrement) or as the average phase difference between the Laplacian of the fundamental harmonic of the Fourier transformed displacement field and the field itself. Hence, the attenuation constant $\alpha$ is given by [25]

$$
\alpha=\kappa \tan (\phi / 2)=\frac{2 \pi}{\lambda} \tan (\phi / 2)
$$

Here, the loss angle $\phi$ is globally defined as $\phi=$ mean $\left\{\angle\left[-\Gamma(\omega) / \nabla^{2} \Gamma(\omega)\right]\right\}$. Local estimates of the wavenumber $\kappa$ were obtained using the LFE implementation provided by Grimm [22]. Regional estimates of the viscoelastic shear modulus were extracted by inverting Eq. (6). As a first approximation, we assume viscous isotropy in this study.

The distortion-based LFE isotropic viscoelastic method was verified by a closed-form solution. Radial wave propagation in a cylindrical geometry was considered in this study. Bessel functions form the basis of the analytical solution. Following Okamoto [20], we simulated the $u_{3}$ wave field; however, to highlight the importance of distortion-based inversion, we add to this solution harmonic rigid body motion at the fundamental frequency (Fig. 1). Inversion of the analytical displacement and distortion fields was performed without the use of either spatial or frequency domain filters.

2.3 Estimation of Transversely Isotropic Material Parameters. Transversely isotropic materials have a single axis of symmetry normal to the plane of isotropy. Fiber-reinforced materials, such as biologic tissues, are often mechanically idealized as transversely isotropic, with the axis of symmetry being the fiber axis. Two different shear moduli govern shear of the plane of isotropy (normal to the fiber axis) and shear of planes containing the fiber axis (see Fig. 2) [26]. To demonstrate the application of LFE to wave propagation in an anisotropic medium, we consider shear waves in a transversely isotropic, incompressible, soft material in a cylindrical solid.

An attractive feature of LFE is that it can be applied to directionally filtered displacement data, which can facilitate anisotropic material property inversion. The process of directionally filtering displacement to isolate specific components (directions) of wave 

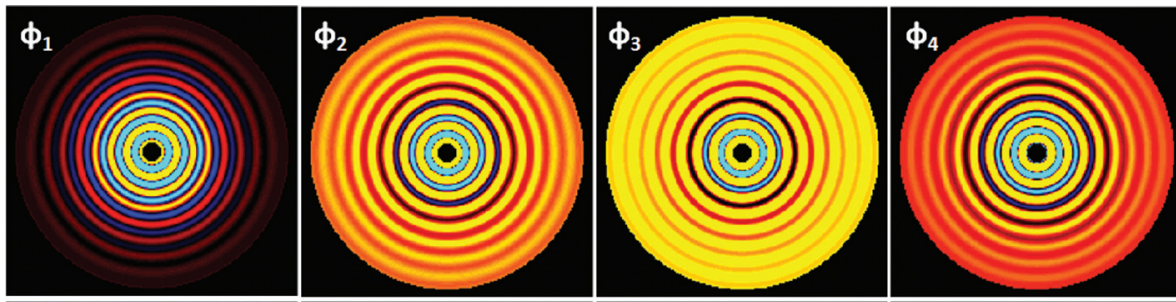

$1 \mu \mathrm{m}$
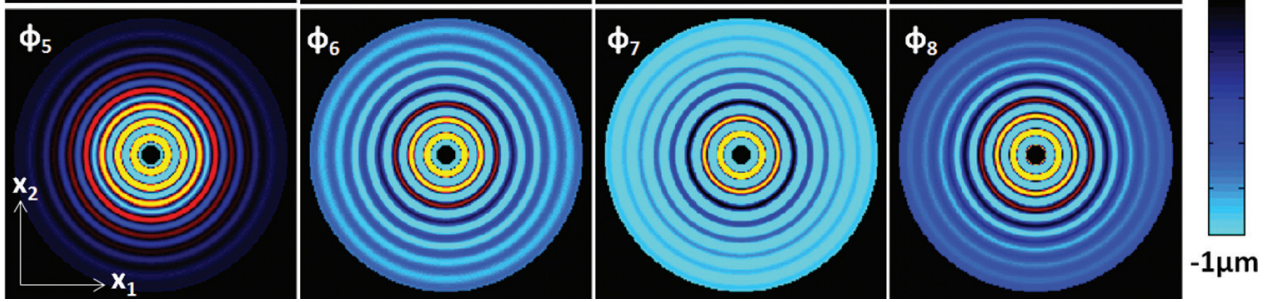

Fig. 1 A time history of the axial displacement field produced by the closed-form analytical solution. The field is shown through a series of eight phase offsets $\left(\phi_{1}, \phi_{2}, \ldots, \phi_{8}\right)$ over one complete cycle. Outwardly propagating shear waves are excited by the axial vibration of a central filament. In addition to the Bessel function solution, a harmonic axial rigid body displacement was added. Parameters: $\mathrm{G}^{\prime}=1.2 \mathrm{kPa}, \mathrm{G}^{\prime \prime}=0.18 \mathrm{kPa}$, density $\rho=1100 \mathrm{~kg} / \mathrm{m}^{3}$, frequency $f=400$ $\mathrm{Hz}$, and cylinder radius $R=22.5 \mathrm{~mm}$.

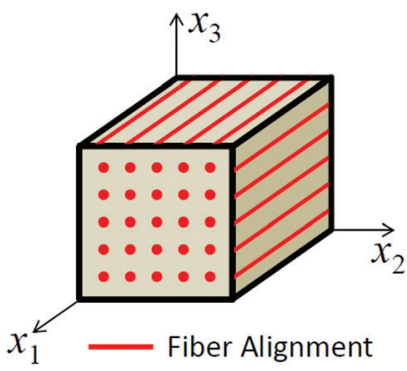

(a)

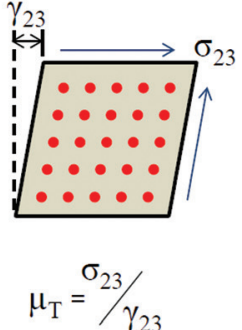

(b)
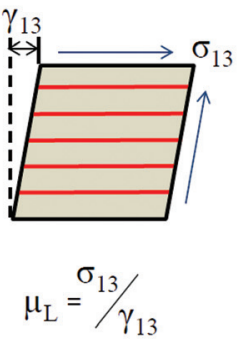

(c)

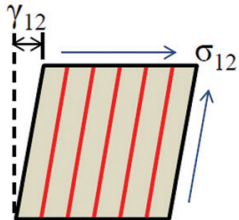

$$
\mu_{\mathrm{L}}=\sigma_{12} / \gamma_{12}
$$

(d)

Fig. 2 (a) Transversely isotropic material model with the axis of symmetry (fiber axis) aligned with the $x_{1}$-axis. $(b)$-(d) Application of shear stress along a face produces a corresponding shear strain in proportion to the shear modulus. In transversely isotropic media there are two shear moduli: $\mu_{T}$ describes shear in a plane normal to the symmetry axis, and $\mu_{L}$ in planes parallel to the symmetry axis. Biological media often exhibit $\mu_{L}>\mu_{T}\left(\gamma_{\mathrm{ij}}=2 \epsilon_{\mathrm{ij}}\right.$, for $\left.i \neq j\right)$. Reproduced from Ref. [26].

propagation has been described and used by others to enhance isotropic material estimates [27]. Direct inversion of MRE displacement fields by fitting to complete wave equations has been used to approximate anisotropic elastic mechanical properties $[28,29]$. The accuracy and robustness of these methods is still a topic of active research. We focus here on a simple LFE-based method and demonstrate its validity for the estimation of anisotropic properties.

In this example, the fiber direction is purely in-plane and aligned with the $x_{1}$-axis of the reference frame (see Fig. 3). Excitation is provided by the harmonic axial motion $u_{0} \cos (\omega t+\phi)$ of a central filament, or analogous harmonic axial displacement of the outer boundary. Steady-state harmonic solutions were obtained with COMSOL Multiphysics finite element software, using the frequency domain solver with 97,038 tetrahedral elements and quadratic interpolation. The solutions were verified by replicating at a coarser mesh resolution. Displacements are almost exclusively out-of-plane: $\overline{\mathbf{u}}=u_{z}(\overline{\mathbf{X}}, t) \overline{\mathbf{e}}_{3}$ where $\overline{\mathbf{X}}=x_{1} \overline{\mathbf{e}}_{1}+x_{2} \overline{\mathbf{e}}_{2}$, so the circumferential component of distortion $\Gamma$ (see Eq. (4)) was analyzed.

Under these conditions, in purely elastic media divergence-free shear waves propagate with speed described by the equation:

$$
\frac{\omega^{2}}{\kappa^{2}} \rho=\mu_{L} \cos ^{2} \theta+\mu_{T} \sin ^{2} \theta
$$

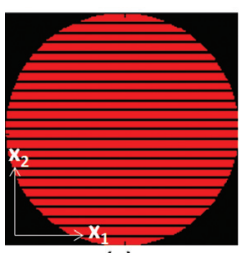

(a)

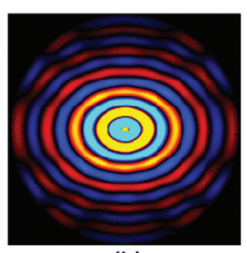

(b)

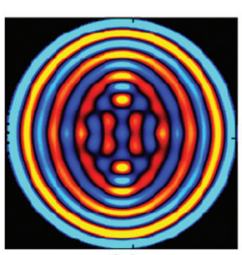

(c) $15 \mu \mathrm{m}$

Fig. 3 Axial displacement fields from finite element simulations. (a) Fiber orientation aligned with the $x_{1}$-axis. (b) Outwardly propagating shear waves excited by the axial vibration of a central filament. (c) Inwardly-propagating waves excited by the axial vibration of the outer boundary. Parameters: shear moduli $\mu_{L}=2000 \mathrm{~Pa}, \mu_{T}=1000 \mathrm{~Pa}$, density $\rho=1000 \mathrm{~kg} / \mathrm{m}^{3}$, frequency $f=5 \mathrm{~Hz}$, and cylinder radius $\mathbf{R}=1 \mathrm{~m}$. The loss factor $\left(\eta=\mathbf{G}^{\prime \prime} / \mathbf{G}^{\prime}\right)$ of the material is $\mathbf{0 . 2 0}$.

In Eq. (8), $\theta$ is the angle between the propagation direction and the fiber axis, $\mu_{L}$ is the shear modulus governing shear deformation of planes parallel to the fiber axis, $\mu_{T}$ is the shear modulus governing shear deformation of the plane normal to the fiber axis, and $\rho$ is the density. 


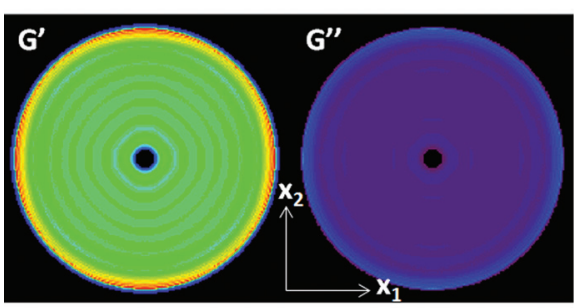

(a)

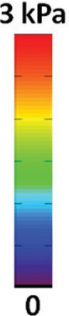

0

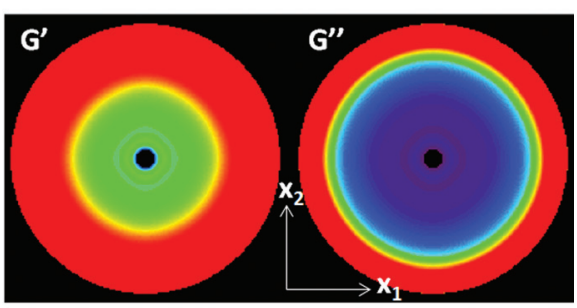

(b)

Fig. 4 Homogeneous isotropic viscoelastic inversion validation in the presence of rigid body (zero strain) motion. Storage $G^{\prime}$ and loss $G^{\prime \prime}$ moduli are shown for inversion of the (a) distortion field $\Gamma\left(G^{\prime}=1.32 \pm 0.37 \mathrm{kPa} ; \mathrm{G}^{\prime \prime}=0.21 \pm 0.09 \mathrm{kPa}\right)$, and $(b)$ displacement field $\left(\mathrm{G}^{\prime}=7.48 \pm 7.78\right.$ $\left.\mathrm{kPa} ; \mathrm{G}^{\prime \prime}=5.55 \pm 9.89 \mathrm{kPa}\right) .\left(\mathrm{G}_{\text {true }}^{\prime}=1.2 \mathrm{kPa} ; \mathrm{G}_{\text {true }}^{\prime \prime}=0.18 \mathrm{kPa}\right.$. $)$

In this example we use LFE to extract the spatial frequencies (wavenumbers) of waves propagating at specific angles, with respect to the fiber direction, and use those wavenumbers and angles in Eq. (8) to estimate the two different shear moduli. To isolate the contributions of plane waves from a particular direction to the signal at a specific location, first the field $\Gamma(\overline{\mathbf{x}}, t)$ is expressed as a Fourier series in terms of harmonic functions of space, each with a different wavenumber, as

$$
\Gamma(\overline{\mathbf{X}}, t)=e^{i \omega t} \sum_{m=-\infty}^{\infty} \sum_{n=-\infty}^{\infty} a_{m n} \exp \left(i \overline{\mathbf{k}}_{\mathbf{m n}} \cdot \overline{\mathbf{X}}\right)+\text { complex conjugate }
$$

where $\overline{\mathbf{k}}_{\mathbf{m n}}=\kappa_{m} \overline{\mathbf{e}}_{\mathbf{1}}+\kappa_{n} \overline{\mathbf{e}}_{\mathbf{2}}$. The angle of propagation $\theta_{m n}$ of each plane wave component is defined as the four-quadrant inverse tangent of $\kappa_{n} / \kappa_{m}$. In practice, only finite numbers of terms $(M$ and $N)$ are used in the Fourier series.

Next, a directional spatial filter defined by

$$
f_{p}\left(\theta_{m n}\right)=\left\{\begin{array}{rr}
\cos ^{2} 4\left(\theta_{m n}-\theta_{p}\right), & \left\|\theta_{m n}-\theta_{p}\right\| \leq \pi / 8 \\
0, & \left\|\theta_{m n}-\theta_{p}\right\|>\pi / 8
\end{array}\right.
$$

is used to extract plane wave components propagating from a sector centered around $\theta_{p}=p \pi / 8$, for 16 sectors $(p=1,2, \ldots 16)$. After inverse Fourier transforming, the filtered data set $\Gamma_{p}(\overline{\mathbf{X}})$ is obtained, Eq. (11) which corresponds to the part of the data explained by propagating waves from the sector centered at $\theta_{p}$, i.e., propagating in the direction $\overline{\mathbf{e}}_{\mathbf{p}}=\cos \theta_{p} \overline{\mathbf{e}}_{\mathbf{1}}+\sin \theta_{p} \overline{\mathbf{e}}_{\mathbf{2}}$.

$$
\Gamma_{p}(\overline{\mathbf{X}})=\sum_{m=-M}^{M} \sum_{n=-N}^{N} f_{p}\left(\theta_{m n}\right) a_{m n} \exp \left(i \overline{\mathbf{k}}_{\mathbf{m n}} \cdot \overline{\mathbf{X}}\right)
$$

Local estimates of anisotropic material parameters are obtained by applying the LFE to the directionally filtered field $\Gamma_{p}(\overline{\mathbf{X}})$. Then, at each location $\overline{\mathbf{X}}$ the spatial frequency $\kappa_{p}(\overline{\mathbf{X}})$ for plane wave propagation in direction $\theta_{p}$ can be estimated. Noting that propagation speed in that direction $c_{p}^{2}=\rho \omega^{2} / \kappa_{p}^{2}$, in principle, we can write Eq. (8) at each location $\overline{\mathbf{X}}$, for all values of $p$ as

$$
\rho\left\{\begin{array}{c}
\omega^{2} / \kappa_{1}^{2} \\
\vdots \\
\omega^{2} / \kappa_{p}^{2}
\end{array}\right\}=\left[\begin{array}{cc}
\cos ^{2} \theta_{1} & \sin ^{2} \theta_{1} \\
\vdots & \vdots \\
\cos ^{2} \theta_{p} & \sin ^{2} \theta_{p}
\end{array}\right]\left\{\begin{array}{l}
\mu_{L} \\
\mu_{T}
\end{array}\right\}, \quad p=1,2, \ldots, 16
$$

Furthermore, we can obtain versions of Eq. (12) for each excitation case. In this example, since we excite both centrally and at the boundary we have 32 potential instances of Eq. (12) which can be simultaneously solved for $\mu_{L}$ and $\mu_{T}$ (in a least squares sense).
While the preceding paragraphs, culminating in Eq. (12), describe the principle of transversely isotropic shear modulus characterization, there are several practical issues to note. Not all 32 instances should be used at a single location, because waves in direction $\theta_{p}$ may not be represented in the signal. Although waves with nonplanar fronts typically contain multiple plane wave components, some directions have negligible contributions. Thus, if the amplitude from a particular direction is lower than a threshold (typically if $\left|\Gamma_{p}(\overline{\mathbf{X}})\right|<0.05|\Gamma|_{\max }$ ), the corresponding instances of Eq. (12) are dropped. The remaining instances of Eq. (12) can be simultaneously solved for each voxel and its nearest neighbors, in order to provide some spatial smoothing and a better conditioned set of equations. If fewer than four instances of Eq. (12) per voxel remain after dropping the low-amplitude directions, no estimates of shear moduli are computed.

The effect of measurement noise on the material parameter estimates was assessed by corrupting simulated displacement data and then inverting these data. Varying extents of measurement noise were simulated by scaling a uniform random distribution of numbers over the interval $[-0.5,0.5]$. Noisy displacement data were generated by combining the simulated measurement noise with analytical displacement field data.

\section{Results}

3.1 Viscoelastic Material Parameters From Analytical Data. LFE inversion of the closed-form wave field highlights the utility of distortion-based material estimates (see Fig. 4). Unlike displacement-based LFE inversion, which contains artifacts induced by rigid body motion, distortion-based material estimates are more uniform. The sensitivity of isotropic material properties to measurement noise is presented in Table 1.

3.2 Viscoelastic Material Parameters From Experimental Data. Practical implementation of the isotropic viscoelastic distortion-based LFE algorithm was verified with published data (see Fig. 5). All raw displacement data acquired in the Okamoto [20] phantom study were reprocessed using the distortion-based LFE algorithm described within. In the Okamoto study, inversion was performed by local total least-squares (TLS) fit over a spatial kernel of $13 \times 13 \times 7$ voxels. The mean value and standard deviation of $G^{\prime}$ and $G^{\prime \prime}$ were reported using only voxels in which the normalized residual error of the local fit was less than 0.5 .

Table 1 Variation in isotropic material properties due to measurement noise. (Actual values: $\mathrm{G}^{\prime}=1.2 \mathrm{kPa}$ and $\mathrm{G}^{\prime \prime}=0.18 \mathrm{kPa}$.)

\begin{tabular}{lcc}
\hline \hline Noise level & $\mathrm{G}^{\prime}(\mathrm{kPa})$ & $\mathrm{G}^{\prime \prime}(\mathrm{kPa})$ \\
\hline 0 & $1.32 \pm 0.37$ & $0.21 \pm 0.09$ \\
$0.25^{*}$ Mean amplitude & $1.15 \pm 0.31$ & $0.17 \pm 0.08$ \\
$0.50^{*}$ Mean amplitude & $1.01 \pm 0.31$ & $0.14 \pm 0.06$ \\
\hline \hline
\end{tabular}



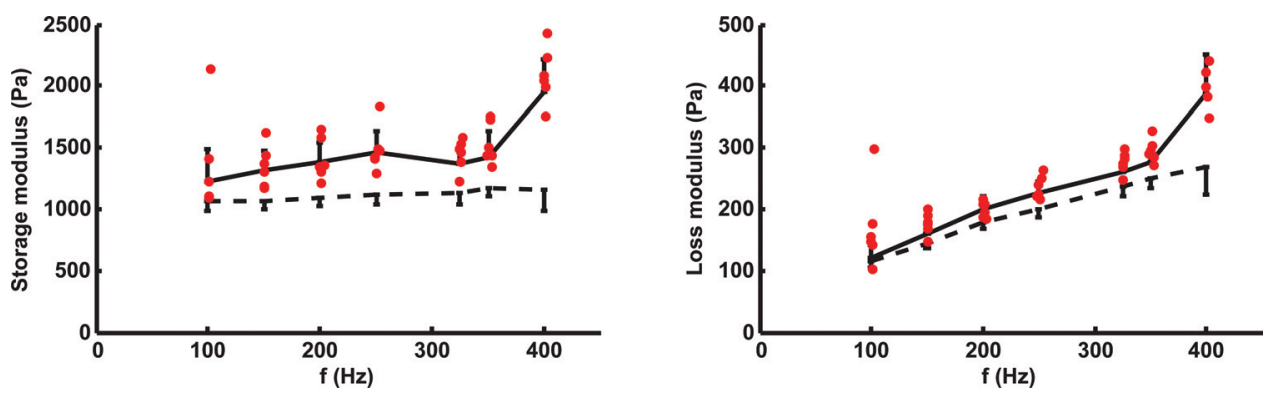

Fig. 5 Comparison of the viscoelastic distortion-based LFE inversion (red dots) with total least-squares (TLS) direct inversion (black lines) performed by Okamoto [20]. Results in this study were not corrected for test-order temperature variations as they were in the Okamoto study (dashed black lines). Error bars represent \pm 1 std. dev.

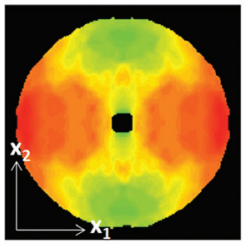

(a)

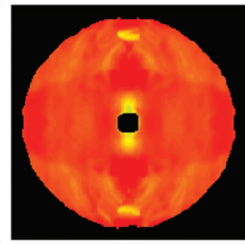

(b)

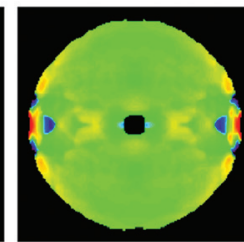

(c)

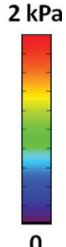

Fig. 6 (a) Estimates of shear modulus assuming an isotropic material model. (b) Estimates of shear modulus $\mu_{L}$ (mean \pm std. dev. = $1761 \pm 111 \mathrm{~Pa}$ ) obtained using the LFE-based approach for transversely isotropic materials. (c) Estimates of shear modulus $\mu_{T}(1045 \pm 152 \mathrm{~Pa})$. The true values are $\mu_{L}=2000$ and $\mu_{T}=1000$. In the anisotropic case, the estimation error increases in regions where the shear wave does not sufficiently activate a plane of shear governed by either $\mu_{L}$ or $\mu_{T}$.

Table 2 Variation in anisotropic material properties due to measurement noise. (Actual values: $\mu_{L}=2000 \mathrm{~Pa}, \mu_{T}=1000 \mathrm{~Pa}$, and $\eta=0.20$.)

\begin{tabular}{lccc}
\hline \hline Noise level & $\mu_{L}(\mathrm{~Pa})$ & $\mu_{T}(\mathrm{~Pa})$ & $\eta$ (Loss factor) \\
\hline 0 & $1761 \pm 111$ & $1045 \pm 152$ & 0.180 \\
$0.25^{*}$ Mean amplitude & $1667 \pm 132$ & $1014 \pm 228$ & 0.162 \\
$0.50^{*}$ Mean amplitude & $1647 \pm 131$ & $1071 \pm 210$ & 0.161 \\
\hline \hline
\end{tabular}

3.3 Anisotropic Material Parameters From Simulated Data. Estimates of the two elastic shear moduli obtained by the LFE of directionally-filtered data from the FE simulations of axial waves in a cylindrical sample are shown in Fig. 6. The corresponding loss moduli (not shown) are estimated simply from $G^{\prime \prime}=\eta G^{\prime}$. The sensitivity of anisotropic material properties to measurement noise is presented in Table 2 .

\section{Discussion and Conclusion}

An extension of the LFE parameter identification method has been verified to handle viscoelastic and anisotropic media. We have reduced the error in our estimates of shear parameters by applying the LFE to distortional fields rather than displacement fields.

The LFE estimates of isotropic storage modulus fall within $10 \%$ of true values (or values measured by direct mechanical test) and loss moduli fall within $20 \%$ of the true values. For transversely isotropic materials, the estimates of both moduli are generally within $10 \%$ of true values, except that the accuracy of estimates is reduced where the wave propagation does not involve the corresponding shear deformation.

The discrepancy between the results presented here and those reported by Okamoto may be attributed to the use of moduli with unquantified certainties. In Fig. 5, each data point corresponds to a different gelatin sample, which may vary slightly in composition and temperature. In addition, there is noise due to measurement error, discretization, and numerical differentiation. Isotropic viscoelastic material estimates using LFE were facilitated by Grimm's wavenumber $\kappa$ extraction implementation [22]. The original LFE image processing implementation [21] includes a measure of certainty for each point in the $\kappa$ field, with the idea that wavenumbers with low certainty should either be rejected or used cautiously. This is useful for assessing the relative confidence on local frequency estimates. In the Okamoto study, which used total least-squares inversion, an error metric was used to reject moduli above a predetermined threshold.

Overall, LFE is confirmed to be powerful and robust method for the estimation of material parameters and can be extended to estimated parameters of viscoelastic and anisotropic materials. The analyses in this study were restricted to $2 \mathrm{D}$. An extension to 3D should be the next step in the development of these techniques. Full 3D displacement fields (i.e., from multiple contiguous image planes) can be readily acquired and, through-image-plane derivatives, are straightforward to perform. Extension of the LFE algorithm to $3 \mathrm{D}$ will enable anisotropic viscoelastic properties from more complicated propagation fields. Use of the correspondence principle limits viscoelastic reconstruction to the linear material response regime.

Boundary conditions will affect the wave field. Constructive and destructive interference of waves can distort the apparent wavelength and, consequently, affect the estimates of the mechanical properties of the shear modulus. However, directionally filtering the displacement or curl field reduces the wave interference. In addition, each directional component of the wave field is used to estimate the shear modulus of the material, reducing the uncertainty attributed to boundary conditions.

The analysis of transversely isotropic materials was greatly simplified by the fact that the shear waves studied here all involved displacements polarized perpendicular to the fiber axis. In the more general scenario, shear waves with displacement components in the direction of the fiber axis will have wavelengths that depend on a third parameter which describes the difference between tensile moduli [30]. We note that to estimate more than one elastic parameter, additional data are required from waves propagating in different directions. Moreover, MRE estimates in heterogeneous media need to be interpreted with caution, especially when the scale of the heterogeneity is similar to or shorter than the wavelength of the dynamic deformation. Uncertainty in modulus estimates of heterogeneous media have been addressed by previous studies $[5,21]$ for the elastic isotropic case.

This inversion method will likely be best suited for cases when the axis of symmetry for the transversely isotropic material is known, or at least estimated with good accuracy. In many anisotropic tissues, the axis of material symmetry (e.g., fiber axis) is aligned with the first principal axis of the diffusion tensor, which can be readily estimated by diffusion tensor imaging. This LFE 
method could be applied to components of the displacement field that propagate in specific directions in a coordinate system aligned with the fiber axis. Consequentially, shear moduli would be estimated in the fiber-referenced coordinate systems and would need to be transformed back into the physical (laboratory) coordinate system.

Measurements of tissue viscoelastic parameters in vivo are much-needed to validate computer models; for example, in simulations of traumatic brain injury. The values of these parameters may also serve as indicators of tissue health or disease [7,13-15]. The current extension of the LFE enhances its utility and further development of this approach is well warranted.

\section{Acknowledgment}

Financial support for this study was provided by NIH Grant No. R01 NS055951 (P.V. Bayly).

\section{References}

[1] Muthupillai, R., Lomas, D. J., Rossman, P. J., Greenleaf, J. F., Manduca, A., and Ehman, R. L., 1995, "Magnetic Resonance Elastography by Direct Visualization of Propagating Acoustic Strain Waves," Science, 269(5232), pp. 1854-1857.

[2] Kruse, S. A., Smith, J. A., Lawrence, A. J., Dresner, M. A., Manduca, A., Greenleaf, J. F., Ehman, R. L., 2000, "Tissue Characterization Using Magnetic Resonance Elastography: Preliminary Results,” Phys. Med. Biol., 45(6), pp.1579-1590.

[3] Sinkus, R., Lorenzen, J., Schrader, D., Lorenzen, M., Dargatz, M., and Holtz, D., 2000, "High-Resolution Tensor MR Elastography for Breast Tumour Detection," Phys. Med. Biol., 45(6), pp. 1649-1664.

[4] Braun, J., Buntkowsky, G., Bernarding, J., Tolxdorff, T., and Sack, I., 2011, "Simulation and Analysis of Magnetic Resonance Elastography Wave Images Using Coupled Harmonic Oscillators and Gaussian Local Frequency Estimation," Magn. Reson. Imaging, 19(5), pp. 703-713.

[5] Manduca, A., Oliphant, T. E., Dresner, M. A., Mahowald, J. L., Kruse, S. A., Amromin, E., Felmlee, J. P., Greenleaf, J. F., Ehman, R. L., 2001, "Magnetic Resonance Elastography: Non-Invasive Mapping of Tissue Elasticity," Med. Image Anal., 5(4), pp. 237-254.

[6] Van Houten, E. E., Paulsen, K. D., Miga, M. I., Kennedy, F. E., and Weaver, J. B., 1999, "An Overlapping Subzone Technique for MR-Based Elastic Property Reconstruction,” Magn. Reson. Med., 42(4), pp. 779-786.

[7] Sinkus, R., Tanter, M., Xydeas, T., Catheline, S., Bercoff, J., and Fink, M., 2005, "Viscoelastic Shear Properties of In Vivo Breast Lesions Measured by MR Elastography," J. Magn. Reson. Imaging, 23(2), pp. 159-165.

[8] Klatt, D., Hamhaber, U., Asbach, P., Braun, J., and Sack, I.,2007, "Noninvasive Assessment of the Rheological Behavior of Human Organs Using Multifrequency MR Elastography: A Study of Brain and Liver Viscoelasticity," Phys. Med. Biol., 52(24), pp. 7281-7294.

[9] Green, M. A., Bilston, L. E., and Sinkus, R., 2008, "In Vivo Brain Viscoelastic Properties Measured by Magnetic Resonance Elastography," NMR Biomed., 21(7), pp. 755-764.

[10] Sack, I., Beierbach, B., Hamhaber, U., Klatt, D., and Braun, J., 2008, "Noninvasive Measurement of Brain Viscoelasticity Using Magnetic Resonance Elastography," NMR Biomed., 21, pp. 265-271.
[11] Sack, I., Rump, J., Elgeti, T., Samani, A., and Braun, J., 2009, "MR Elastography of the Human Heart: Noninvasive Assessment of Myocardial Elasticity Changes By Shear Wave Amplitude Variations," Magn. Reson. Med., 61(3), pp. 668-677.

[12] Klatt, D., Papazoglou, S., Braun, J., and Sack, I., 2010, "Viscoelasticity-Based MR Elastography of Skeletal Muscle,” Phys. Med. Biol., 55, pp. 6445-6459.

[13] Streitberger, K. J., Wiener, E., Hoffmann, J., Freimann, F. B., Klatt, D., Braun J., Lin, K., McLaughlin, J., Sprung, C., Klingebiel, R., Sack, I., 2010, "In Vivo Viscoelastic Properties of the Brain in Normal Pressure Hydrocephalus," NMR Biomed., 24(7), pp. 385-392.

[14] Wuerfel, J., Paul, F., Beierbach, B., Hamhaber, U., Klatt, D., Papazoglou, S., Zipp, F., Martus, P., Braun, J., Sack, I., 2010, "MR-Elastography Reveals Degradation of Tissue Integrity in Multiple Sclerosis," Neuroimage, 49(3), pp. $2520-2525$

[15] Clayton, E. H., Garbow, J. R., and Bayly, P. V., 2011, "Frequency-Dependent Viscoelastic Parameters of Mouse Brain Tissue Estimated by MR Elastography," Phys. Med. Biol., 56(8), pp. 2391-2406.

[16] Riek, K., Klatt, D., Nuzha, H., Mueller, S., Neumann, U., Sack, I., Braun, J., 2011, "Wide-Range Dynamic Magnetic Resonance Elastography," J. Biomech. 44(7), pp. 1380-1386.

[17] Clayton, E. H., Genin, G. M., and Bayly, P. V., 2012, "Transmission, Attenuation and Reflection of Shear Waves in the Human Brain," J. R. Soc., Interface, 76, pp. 2899-2910.

[18] Chatelin, S., Constantinesco, A., and Willinger, R., 2010, "Fifty Years of Brain Tissue Mechanical Testing: From In Vitro to In Vivo Investigations," Biorheology, 47(5-6), pp. 255-276.

[19] Whitham, G. B., 1974, Linear and Nonlinear Waves, Wiley, New York.

[20] Okamoto, R. J., Clayton, E. H., and Bayly, P. V., 2011, "Viscoelastic Properties of Soft Gels: Comparison of Magnetic Resonance Elastography and Dynamic Shear Testing in the Shear Wave Regime," Phys. Med. Biol., 56(19), pp. 6379-6400.

[21] Knutsson, H., Westin, C. F., and Granlund, G. H., 1994, "Local Multiscale Frequency and Bandwidth Estimation," Proceedings of the IEEE International Conference on Image Processing, pp. 36-40.

[22] Grimm, R. C., Lake, D. S., Manduca, A., Ehman, R. L., 2006, MRE/Wave version: 1 July 2006, Mayo Clinic, http://mayoresearch.mayo.edu/mayo/research/ ehman lab/mrw-wave.cfm

[23] Kolsky, H., 1963, Stress Waves in Solids, Dover, New York.

[24] Auld, B. A., 1990, Acoustic Fields and Waves in Solids, R.E. Krieger, Malabar, FL.

[25] Christensen, R. M., 2005, Mechanics of Composite Materials, Dover, New York.

[26] Clayton, E. H., 2012, "Magnetic Resonance Elastography of the Brain: From Phantom to Mouse to Man," Ph.D. dissertation, Department of Mechanical Engineering and Materials Science, Washington University in St. Louis, St. Louis.

[27] Manduca, A., Lake, D. S., Kruse, S. A., and Ehman, R. L., 2003, "Spatio-Temporal Directional Filtering for Improved Inversion of MR Elastography Images," Med. Image Anal., 7(4), pp. 465-73.

[28] Sinkus, R., Tanter, M., Catheline, S., Lorenzen, J., Kuhl, C., Sondermann, E., Fink, M., 2005, "Imaging Anisotropic and Viscous Properties of Breast Tissue by Magnetic Resonance Elastography," Magn. Reson. Med., 53(2), pp. 372-387.

[29] Romano, A. J., Scheel, M., Hirsch, S., Braun, J., and Sack, I., 2012, "In Vivo Waveguide Elastography of White Matter Tracts in the Human Brain," Magn. Reson. Med. (epub ahead of print).

[30] Spencer, A. J. M., 1984, Continuum Theory of the Mechanics of FibreReinforced Composites, Springer-Verlag, Berlin. 